\title{
ON AN ANALYTIC SIMPLIFICATION OF A SYSTEM OF LINEAR ORDINARY DIFFERENTIAL EQUATIONS CONTAINING A PARAMETER ${ }^{1}$
}

\author{
PO-FANG HSIEH
}

1. Introduction. Let $x$ be a complex variable and let $D_{0}$ be a simply connected compact domain in the $x$-plane which contains the origin $O$ in its interior. Let $\rho_{0}$ be a positive number. Consider a system of linear ordinary differential equations of the form

$$
\epsilon^{\sigma} d y / d x=A(x, \epsilon) y
$$

where $\sigma$ is a nonnegative integer, $\epsilon$ is a complex parameter, $y$ is an $n$-dimensional vector, and $A(x, \epsilon)$ is an $n$ by $n$ matrix with components holomorphic in a domain

$$
x \in D_{0}, \quad|\epsilon| \leqq \rho_{0} .
$$

Let

$$
A(x, \epsilon)=\sum_{k=0}^{\infty} \epsilon^{k} A_{k}(x)
$$

be the expansion of $A(x, \epsilon)$ in powers of $\epsilon$, where $A_{k}(x)$ are holomorphic in $D_{0}$.

In this paper, we shall prove the following theorem.

TheOREM. For each nonnegative integer $m$, there exists an $n$ by $n$ matrix $P(x, \epsilon)$ satisfying the following conditions:

(i) the components of $P(x, \epsilon)$ are holomorphic with respect to $(x, \epsilon)$ in the domain

$$
x \in D_{1}, \quad|\epsilon| \leqq \rho_{0},
$$

where $D_{1}$ is a certain subdomain of $D_{0}$ which contains the origin $O$ in its interior;

(ii) $P(x, 0)=1_{n}$ for $x \in D_{1}$ and $P(0, \epsilon)=1_{n}$ for $|\epsilon| \leqq \rho_{0}$, where $1_{n}$ is the $n$ by $n$ unit-matrix;

(iii) the system (1.1) is reduced to

$$
\epsilon^{\sigma} d u / d x=\left\{\sum_{k=0}^{m} \epsilon^{k} A_{k}(x)+\epsilon^{m+1} \sum_{k=0}^{\sigma-1} \epsilon^{k} B_{k}(x)\right\} u
$$

Received by the editors May 10, 1967.

1 This paper was partially supported by a Faculty Research Fellowship at Western Michigan University and by NSF Grant GP-7862. 
by the transformation

$$
y=P(x, \epsilon) u
$$

where $B_{k}(x)(k=0,1, \cdots, \sigma-1)$ are $n$ by $n$ matrices whose components are holomorphic for $x \in D_{1}$.

REMARK. In case when $\sigma=1$, the right-hand member of (1.4) has the form

$$
\left\{\sum_{k=0}^{m} \epsilon^{k} A_{k}(x)+\epsilon^{m+1} B_{0}(x)\right\} u .
$$

In particular if $\sigma=1$ and $m=0$, the system (1.4) has the form

$$
\epsilon d u / d x=\left\{A_{0}(x)+\epsilon B_{0}(x)\right\} u .
$$

G. D. Birkhoff [2] has proved a result similar to ours for linear differential equations at an irregular singular point. Since his result was concerned with the behavior of solutions at a singular point with respect to the independent variable, it was necessary to assume a certain condition on the monodromy matrix at the singular point. (See, for example, H. L. Turrittin [5].) We do not need to assume such a condition, insomuch as our result is only concerned with the singularity with respect to the parameter.

It might be possible to prove our theorem by using a method similar to that of Birkhoff's result. However, it is necessary to modify his lemma on matrices [1] in such a manner that this lemma can be used for matrices depending on many variables. Instead of doing this, we shall prove our theorem by using a direct method which is based on the theory of ordinary differential equations in a Banach space. This method was suggested by Y. Sibuya in one of his papers [4]. The author is indebted to Professor Yasutaka Sibuya for valuable discussions during this work.

2. Fundamental nonlinear equations. Let us put

$$
P(x, \epsilon)=1_{n}+\epsilon^{m+1} \sum_{k=0}^{\infty} \epsilon^{k} P_{k}(x)
$$

and

$$
B(x, \epsilon)=\sum_{k=0}^{m+\sigma} \epsilon^{k} B_{k}(x)
$$

where 


$$
\begin{aligned}
\hat{B}_{k}(x) & =A_{k}(x), \quad(k=0,1, \cdots, m), \\
& =B_{k-m-1}(x), \quad(k=m+1, m+2, \cdots, m+\sigma) .
\end{aligned}
$$

In order that the transformation (1.5) reduces the system (1.1) to the system (1.4), we must have the differential equation

$$
\epsilon^{\sigma} d P / d x=A(x, \epsilon) P-P B
$$

satisfied by the matrices $P$ and $B$. From this equation we derive

$$
\begin{aligned}
& 0=A_{m+1+k}(x)-\hat{B}_{m+1+k}(x) \\
& +\sum_{h=0}^{k}\left\{A_{k-h}(x) P_{h}(x)-P_{h}(x) \hat{B}_{k-h}(x)\right\}, \\
& \quad(k=0,1, \cdots, \sigma-1)
\end{aligned}
$$

and

$$
\begin{aligned}
d P_{k}(x) / d x= & A_{m+1+\sigma+k}(x)+\sum_{h=0}^{\sigma+k} A_{\sigma+k-h}(x) P_{h}(x) \\
& -\sum_{h=k-m}^{\sigma+k} P_{h}(x) \hat{B}_{\sigma+k-h}(x), \quad(k=0,1,2, \cdots),
\end{aligned}
$$

where

$$
P_{h}(x) \equiv 0 \quad \text { if } \quad h<0 .
$$

We shall determine $P$ and $B$ by solving these equations.

We should remark here that, in many cases, formal power series $P$ and $B$ which satisfy the equation (2.4) are not convergent. (See, for example, Y. Sibuya [3] and W. Wasow [6].) In order to get $P$ as a convergent power series in $\epsilon$ we must choose a suitable $B$. To do this, first of all, let us solve (2.5) with respect to $\hat{B}_{m+1+k}(x)$. Then we get

$$
\begin{array}{r}
\hat{B}_{m+1+k}(x)=A_{m+1+k}(x)+H_{m+1+k}\left(x ; P_{0}, \cdots, P_{k}\right), \\
(k=0,1,2, \cdots, \sigma-1)
\end{array}
$$

where $H_{j}$ are defined by

$$
\begin{aligned}
H_{j}=0, \quad(j=0,1, \cdots, m), & \\
H_{m+1+k}\left(x ; P_{0}, \cdots, P_{k}\right)= & \sum_{h=0}^{k}\left\{A_{k-h}(x) P_{h}-P_{h} A_{k-h}(x)\right\} \\
& -\sum_{h=0}^{k} P_{h} H_{k-h}, \\
& (k=0,1, \cdots, \sigma-1) .
\end{aligned}
$$


Substituting (2.8) into (2.6) we get

$$
d P_{k} / d x=f_{k}(x ; \mathfrak{B}), \quad(k=0,1,2, \cdots),
$$

where

$$
\begin{aligned}
f_{k}(x ; \mathfrak{P})= & A_{m+1+\sigma+k}(x)+\sum_{h=0}^{\sigma+k} A_{\sigma+k-h}(x) P_{h} \\
& -\sum_{h=k-m}^{\sigma+k} P_{h} A_{\sigma+k-h}(x)-\sum_{h=k-m}^{\sigma+k} P_{h} H_{\sigma+k-h}(x ; \mathfrak{B}), \\
& \quad(k=0,1,2, \cdots),
\end{aligned}
$$

with $\mathfrak{B}$ denoting an infinite-dimensional vector $\left\{P_{k} ; k=0,1, \cdots\right\}$. If we denote by $\mathfrak{f}(x ; \mathfrak{B})$ the infinite-dimensional vector

$$
\left\{f_{k}(x ; \mathfrak{B}) ; \quad k=0,1,2, \cdots\right\},
$$

then equations (2.11) can be written in the form

$$
d \mathfrak{P} / d x=\mathfrak{f}(x ; \mathfrak{B}) .
$$

We shall solve this differential equation in a suitable Banach space. If $\mathfrak{B}$ is determined, then the quantities $B_{k}$ are determined by (2.8) and (2.9).

3. A lemma on $\mathrm{f}(x ; \mathfrak{B})$. Since components of the matrix $A(x, \epsilon)$ are holomorphic in the domain (1.2), there is a positive number $\rho$ such that $\rho>\rho_{0}$ and that components of $A$ are holomorphic in the domain

$$
x \in D_{0}, \quad|\epsilon| \leqq \rho .
$$

Let us denote by $\mathfrak{B}$ the set of all infinite-dimensional vectors $\mathfrak{P}$ $=\left\{P_{k} ; k=0,1,2, \cdots\right\}$ such that

(i) $P_{k}$ are $n$ by $n$ matrices whose components are complex numbers;

(ii) $\sum_{k=0}^{\infty} \rho^{k}\left|P_{k}\right|<+\infty$,

where $\left|P_{k}\right|$ is the sum of absolute values of components of $P_{k}$. For each $\mathfrak{P}$, let us define a norm $\|\mathfrak{P}\|$ by

$$
\|\mathfrak{P}\|=\sum_{k=0}^{\infty} \rho^{k}\left|P_{k}\right| .
$$

Then we can regard $\mathfrak{B}$ as a Banach space over the field of complex numbers.

Let $\mathfrak{B}(x)$ be a mapping from $D_{0}$ to $\mathfrak{B}$. This mapping is said to be $\mathfrak{B}$-holomorphic in $D_{0}$ if there exists another mapping $\mathfrak{Q}(x)$ from $D_{0}$ to $\mathfrak{B}$ such that 


$$
\lim _{h \rightarrow 0}\left\|h^{-1}\{\mathfrak{P}(x+h)-\mathfrak{B}(x)\}-\mathfrak{Q}(x)\right\|=0
$$

for all $x \in D_{0}$. Wedenote $\mathfrak{Q}$ by $d \mathfrak{B} / d x$. If $\mathfrak{B}(x)=\left\{P_{k}(x) ; k=0,1,2, \cdots\right\}$ is $\mathfrak{B}$-holomorphic in $D_{0}$, then each matrix $P_{k}(x)$ is holomorphic in $D_{0}$ and

$$
d \mathfrak{B}(x) / d x=\left\{d P_{k}(x) / d x ; k=0,1,2, \cdots\right\} .
$$

Now we can prove the following lemma.

Lemma. Let $\mathfrak{f}(x, \mathfrak{B})$ be the infinite-dimensional vector whose components $f_{k}(x ; \mathfrak{B})$ are given by $(2.11)$. Then $\mathfrak{f}(x ; \mathfrak{B})$ is a mapping from $D_{0} \times \mathfrak{B}$ to $\mathfrak{B}$ which has the following properties:

(i) for each positive number $R$ there are two positive numbers $G(R)$ and $K(R)$ such that

$$
\|\mathfrak{f}(x ; \mathfrak{B})\| \leqq G(R) \quad \text { for }\|\mathfrak{P}\| \leqq R
$$

and

$$
\|\mathfrak{f}(x ; \mathfrak{B})-\mathfrak{f}(x ; \tilde{\mathfrak{P}})\| \leqq K(R)\|\mathfrak{P}-\tilde{\mathfrak{P}}\| \quad \text { for }\|\mathfrak{P}\| \leqq R,\|\tilde{\mathfrak{P}}\| \leqq R
$$

(ii) $\mathfrak{f}(x ; \mathfrak{B}(x))$ is $\mathfrak{B}$-holomorphic in $D_{0}$ if $\mathfrak{B}(x)$ is $\mathfrak{B}$-holomorphic in $D_{0}$.

Let us consider a formal power series in $\epsilon$ which is defined by

$$
F(x, \mathfrak{P}, \epsilon)=\sum_{k=0}^{\infty} \epsilon^{k} f_{k}(x ; \mathfrak{P}) .
$$

Then from the definition of $f_{k}$ we derive the following formal identity:

$$
\begin{array}{r}
F(x, \mathfrak{P}, \epsilon)=\sum_{k=0}^{\infty} \epsilon^{k} A_{m+1+\sigma+k}(x)+\frac{1}{\epsilon^{\sigma}}\left\{\sum_{k=0}^{\infty} \epsilon^{k} A_{k}(x) \sum_{k=0}^{\infty} \epsilon^{k} P_{k}\right. \\
\left.-\sum_{k=0}^{\sigma-1} \epsilon^{k} \sum_{h=0}^{k} A_{k-h}(x) P_{h}\right\} \\
-\frac{1}{\epsilon^{\sigma}}\left\{\sum_{k=0}^{\infty} \epsilon^{k} P_{k} \sum_{k=0}^{m+\sigma} \epsilon^{k}\left[A_{k}(x)+B_{k}(x ; \mathfrak{B})\right]\right. \\
\left.-\sum_{k=0}^{\sigma-1} \epsilon^{k} \sum_{h=0}^{k} P_{h}\left[A_{k-h}(x)+H_{k-h}(x ; \mathfrak{P})\right]\right\} .
\end{array}
$$

By using (3.7), we can prove the Lemma in a straightforward manner.

4. Proof of Theorem. We shall construct the matrix $P(x, \epsilon)$ by solving the differential equation (2.12) with the initial condition 


$$
\mathfrak{B}(0)=0 .
$$

To do this, we use the method of successive approximations. By virtue of the Lemma of $\S 3$, we can construct, in this manner, a unique solution $\mathfrak{B}(x)$ which is $\mathfrak{B}$-holomorphic in a subdomain $D_{1}$ of $D_{0}$ which contains 0 in its interior. Since $d \mathfrak{B}(x) / d x$ is given by (3.4), the solution $\mathfrak{B}(x)$ gives the desired matrix $P(x, \epsilon)$. The matrix $B(x, \epsilon)$ is determined by (2.8) and (2.9). This completes the proof of our theorem.

\section{REFERENCES}

1. G. D. Birkhoff, "A theorem on matrices of analytic functions" pp. 240-251 in Collected mathematical papers, Vol. I, Amer. Math. Soc., Providence, R. I., 1950.

2. - "Equivalent singular points of ordinary differential equations," pp. 252-257 in Collected mathematical papers, Vol. I, Amer. Math. Soc., Providence, R.I., 1950.

3. Y. Sibuya, Sur réduction analytique d'un système d'équations différentielles ordinaires linéaires contenant un paramètre, J. Fac. Sci. Univ. Tokyo Sect. I 7 (1958), 527-540.

4. - - On the convergence of formal solutions of systems of linear ordinary differential equations containing a parameter, MRC Tech. Sum. Report \#511, September, 1964.

5. H. L. Turrittin, Reduction of ordinary differential equations to the Birkhoff canonical form, Trans. Amer. Math. Soc. 107 (1963), 485-507.

6. W. Wasow, Asymptotic expansions for ordinary differential equations, Wiley, New York, 1965.

WESTERN Michigan University 